\title{
EDITORIAL
}

\section{IN THE MAY 2009 ISSUE OF CLINICS}

\author{
Mauricio Rocha-e-Silva, Editor
}

doi: $10.1590 / \mathrm{S} 1807-59322009000500001$

In this issue of CLINICS, we wish to highlight a project in which Erbayrak et al. investigated the role of Fecal Calprotectin in evaluating inflammatory bowel disease activity of 65 affected patients versus 20 outpatients as controls. Fecal Calprotectin was found to be is strongly associated with colorectal inflammation indicating organic disease, while all the patients in the control group had a fecal calprotectin value lower than the cut-off point $(50 \mathrm{mg} / \mathrm{kg})$. Because Fecal Calprotectin is a simple and non-invasive method for assessing excretion of macrophages into the gut lumen they claim that it can be used to evaluate the response to treatment, to screen asymptomatic patients, and to predict inflammatory bowel disease relapses.

Correia-Deur et al. documented the phenotypes observed in 26 patients with apparent s-medullary thyroid carcinoma cases to attempt to improve earlier diagnoses of this type of carcinoma. Clinical data was reviewed and peripheral blood was collected and screened for RET germline mutations. Calcitonin levels were increased in all cases. After total thyroidectomy associated with extensive cervical lymph node resection, calcitonin values remained low for at least 12 months in eight of the cases (30.8\%). Routine thyroid cytology yielded disappointing diagnostic accuracy (46.7\%). None of the 26 cases presented germline mutations in the classical hotspots of the RET proto-oncogene. They conclude that cases were identified late. The basal calcitonin measurements and immunostaining for calcitonin were highly useful for diagnosing s-medullary thyroid carcinoma. The rate of complete patient recovery was low, and none of the parameters analyzed were useful predictors of the thyroid tumor size. Our findings support previous recommendations for routine serum calcitonin evaluation and immunostaining analysis involving single thyroid nodules.

Iglezias et al. determined the degree of risk produced by the association of other surgical procedures with surgical

Hospital das Clínicas, Faculdade de Medicina da Universidade de São Paulo - São Paulo/SP, Brazil.

mrsilva36@hcnet.usp.br myocardial revascularization in octogenarian patients and identified the risk factors that best explain hospital mortality. Patients were stratified into revascularized with and without associated procedures. They conclude that a procedure associated with the surgical myocardial revascularization produces an absolute increase in hospital mortality risk of $45 \%$. Variables that contributed to hospital mortality were preoperative endocarditis, preoperative cardiogenic shock, the use of extracorporeal circulation, the length of time of extracorporeal circulation, postoperative creatinine level, and postoperative need for prolonged respiratory support.

De Oliveira et al. evaluated 60 patients treated for idiopathic chronic testicular pain. Priority was to give clinical treatment, which evolved from simple to more complex measures. Microsurgical treatment was performed on those who experienced no considerable pain relief (10 individuals in our study). They found that over a twenty-four-month follow-up period, $70 \%$ of patients showed complete remission and $20 \%$ exhibited partial relief from pain.

Bitencourt et al. report on the applications of radioguided surgery in five oncological cases (excluding those related to breast cancer). This has been widely used to identify the sentinel lymph node and occult lesions in patients with breast cancer. However, few studies have reported the use of this technique for non-breast cancer pathologies. The patients presented with recurrence of renal cell carcinoma, cervical recurrence of papillary carcinoma of the thyroid, recurrence of retroperitoneal sarcoma, lesions of the popliteal fossa, and recurrence of rhabdomyosarcoma of a thigh. In each case, the lesions that were marked preoperatively were ultimately successfully excised. They conclude that the procedure allows for more conservative excisions and reduces the surgery-related morbidity.

Rieder et al. investigated the the feasibility and the cardiorespiratory effects of using positive expiratory airway pressure, a physiotherapeutic tool, in comparison with a T-tube, to wean patients from mechanical ventilation. The two procedures were tested in forty patients who met weaning criteria and had been mechanically-ventilated for more 
than 48 hours, mean age 59 years, including 23 males. They claim that weaning patients from mechanical ventilation with the use of a fixed level of Expiratory Positive Airway Pressure caused an increase in work of breathing that was not accompanied by any other significant cardiorespiratory changes, and suggest that caution must be exercised when using Expiratory Positive Airway Pressure during weaning from mechanical ventilation.

Vaisbich et al. endeavored to characterize clinical, laboratory and genetic parameters of five boys with Nephrogenic diabetes insipidus first diagnosed at 14.6 months (range 6 to 24) and followed for 12.2 years (range 7.8 to 19). Geometric means of the z-scores of weight and stature were -4.5 and -3.6 , at diagnosis and -0.3 and -0.9 , respectively, at the last medical appointment. Mutations in the AVPR2 gene were found in all patients, and the carrier status was confirmed in four of five cases. Two unrelated children presented identical mutations (S167L) in arginine vasopressin R2. Two of the patients had a mutation that was already described in other Brazilian families (R337X), and one patient showed a de novo mutation (Y128D) in arginine vasopressin R2, since his mother's molecular analysis was normal. They claim that the clinical and laboratory characterization of Nephrogenic diabetes insipidus is of paramount importance for disease diagnosis and genetic counseling.

De Lima et al. studied the effect of metformin, when administered to first-degree relatives of type 2 diabetes mellitus subjects who have metabolic syndrome and normal glucose tolerance, to determine whether it could improve the cardiovascular risk profile and reduce the levels of both C-reactive protein and fibrinogen. Thirty-one non-diabetic first-degree relatives of type 2 diabetes mellitus subjects with metabolic syndrome were randomized $(1: 1)$ and double-blinded for placement in the placebo and metformin groups, and conclude that the treatment given to first-degree relatives of type 2 diabetes mellitus sufferers who have metabolic syndrome and normal glucose tolerance improved the cardiovascular risk profile without changing the levels of $\mathrm{C}$-reactive protein and fibrinogen.

Bonanomi et al. retrospectively reviewed the records of 28 new cases of retinoblastoma, 15 of which were unilateral and 13 of which were bilateral, diagnosed between 2003 and 2005 (mean age 33.8 months for unilateral cases, and 19.15 months for bilateral cases) and conclude that in this population, retinoblastoma was diagnosed too late and most eyes were consequently enucleated. In cases with bilateral disease, half of the eyes were preserved.

Andrade et al. compared the metabolic, hemodynamic, autonomic, and endothelial responses to short-term red wine consumption in subjects with hypercholesterolemia $(\mathrm{n}=10)$ or arterial hypertension $(n=9)$, and healthy controls $(n=$ 7), and found that red wine enhanced muscle sympathetic fibular nerve activity in hypercholesterolemic and hypertensive patients, but not in controls. At baseline, brachial artery flow-mediated dilation was impaired in patients with hypercholesterolemia and arterial hypertension; red wine restored the dilation in the hypercholesterolemic group but not in the hypertensive group. They claim that red wine elicits different metabolic, autonomic, and endothelial responses among individuals with hypercholesterolemia or arterial hypertension and healthy controls. The findings highlight the need to consider patient characteristics when evaluating the response to red wine.

Aikawa et al. evaluated the effect of mechanical ventilation with positive end expiratory pressure (PEEP) on mesenteric leukocyte-endothelial interactions in 44 male Wistar rats. Rats were exposed to NO PEEP or to PEEP at 0,5 , and $10 \mathrm{~cm} \mathrm{H}_{2} \mathrm{O}$. After two hours of mechanical ventilation rolling, sticking and migrated leukocytes were significantly increased at a PEEP of $10 \mathrm{~cm} \mathrm{H}_{2} \mathrm{O}$. They conclude that the highest level of intrathoracic pressure was harmful to mesenteric microcirculation in this experimental model of rats with normal lungs and stable systemic blood pressure, a finding that may have relevance for complications related to mechanical ventilation.

Monaco et al. give us a fascinating computer demonstration of the evolution of aging and its implications for medical practice. They stress that the discussion regarding the evolution of aging is almost as old as Darwinian Evolution Theory, but to date, it has remained one of biology's unresolved problems. One issue is how to reconcile natural selection, which is understood as a process that purges deleterious characteristics, with senescence, which seems to offer no advantages to the individual. The two popular explanations for the existence of senescence, namely, (1) the removal of elders for the benefit of the species and (2) the progressive deterioration of the organic machine due to continuous use are described as incorrect. They claim that it is important that the physician understand that senescence, defined as the progressive impairment of an organism, does not necessarily accompany aging, defined as the mere passage of time. The concepts of aging (the unavoidable passage of time), senescence (progressive physiologic impairment), and senility (the pathological development of diseases), are discussed.

We also publish 2 review articles, one by Leão et al. on the Oral complications of HIV disease, one by Landim et al. on Asymmetric dimethylarginine and endothelial dysfunction, and 4 case reports. 